\title{
Somatosensory Pulsatile Tinnitus Syndrome (SSPT) Revisited
}

Robert Aaron Levine*

\begin{abstract}
Introduction: Our initial study reported consecutive patients with constant cardiac synchronous subjective tinnitus (pulsatile tinnitus without an identifiable acoustic source $(\mathrm{P})$ ) all of whom could suppress their pulsations with head and neck intense muscle contractions ("somatic testing" (ST)). The term somatosensory pulsatile tinnitus syndrome (SSPT) was coined to refer to this type of $\mathrm{P}$. With now more than a decade of clinical experience with $\mathrm{P}$, herein are reported (a) other ways $\mathrm{P}$ can present, beside SSPT, (b) how $P$ is related to the somatosensory system and recumbency, and (c) what treatments have been effective.

Methods: Retrospective case series of 58 adults with $\mathrm{P}$ encountered in an outpatient clinic or through telemedicine

Results: P could be constant or intermittent, with or without non-pulsatile tinnitus (nP). $90 \%$ of cases could suppress their pulsations with ST; $9 \%$ could not. In 7 of 11 cases that had no P at time of testing, ST elicited P. The most common type of P was SSPT (constant pulsatile tinnitus suppressible by ST) (60\%). Treatment of head and neck muscle dysfunction (muscle dry needling and Botulinum toxin injection) has abolished P; auricular electrical stimulation was effective in 2 cases.

Conclusion: Suppression of pulsations by ST, eliciting $\mathrm{P}$ by ST, and abolishment of $\mathrm{P}$ by head and neck muscle treatments all support a major role of the craniocervical somatosensory system in the etiology of most, if not all, cases of $P$. Three mechanisms are proposed: (A) somatosensory afferents causing dysfunction of the CNS mechanisms that normally suppress self-generated cardiac and vascular sounds, (B) cardiac synchronous disinhibition of the auditory CNS by somatosensory afferents and (C) some combination of $A$ and $B$.
\end{abstract}

Keywords: Dorsal cochlear nucleus, dry needling, recumbency, somatic testing, TENS, auditory nerve vascular compression. 


\section{INTRODUCTION}

Pulsatile, cardiac-synchronous tinnitus can be objective or subjective and can have several causes. Objective pulsatile tinnitus is a somatosound; it has an acoustic source, causing the pulsatile tinnitus. The somatosound can be related to (a) abnormalities of intracranial or cervical blood flow, (b) defects in the barrier acoustically isolating the cochlea from intracranial blood flow, or (c) aberrant blood flow in the middle ear or cochlea capsule. In all these case it is nearly always unilateral and can often be heard and/or recorded ${ }^{1}$. Subjective pulsatile cardiacsynchronous tinnitus $(P)$ is a phantom sound; it has no recordable or identifiable acoustic source via physical examination, blood work, imaging, or lumbar puncture.

The only well-established cause for lateralized $P$ is auditory nerve vascular compression (VC). Two reports describe abolishment of lateralized $P$ by surgical vascular decompression ${ }^{2,3}$. Four such $\mathrm{P}$ cases were permanently abolished by surgical decompression and every patient described their $\mathrm{P}$ as low-pitched. Five other patients had continuous or monotonous tinnitus that was nonpulsatile (nP) and was abolished by surgery in four, including the only one with low-pitched tinnitus. Nonlateralized $\mathrm{P}$ does not respond to such surgery ${ }^{4}$. VC can also cause paroxysmal staccato "typewriter" tinnitus that is pathognomonic for VC and abolished by surgical decompression ${ }^{5}$. Typewriter tinnitus nearly always can be suppressed by medication ${ }^{6}$.

In evaluating thirteen consecutive $P$ cases, all could suppress their pulsations with somatic testing (ST): intensely activating head and neck muscles ${ }^{7}$. The term "somatosensory pulsatile tinnitus syndrome" (SSPT) was coined, to highlight the connection between activation of the head and neck somatosensory system and P. Further support for this connection came from another patient with left intermittent $P$, who could suppress her pulsations with right jaw deviation and elicit $P$ with other head and neck maneuvers.

With this as background the characteristics of 58 more consecutive $P$ patients, who have been evaluated since our original report are presented. This series provides a broader picture of the heterogeneity of $\mathrm{P}$, beyond SSPT. It includes, on the one hand, (a) some who cannot suppress their pulsations with ST, (b) others whose P is intermittent but can be elicited by ST, and (c) some whose intermittent $P$ is related to recumbency. Included is our experience with treatment trials, balloon occlusion angiography and intra-arterial, intracranial amobarbital infusion.

\section{MATERIALS AND METHODS}

Of the 58 (34 F, 24 M; ages 38 to 82, mean 59) 44 were personally evaluated by the author as part of their patient care. For this reason ethics approval was not required; likewise for those evaluated remotely. 14 were evaluated remotely (telemedicine) through questionnaire, telephone and video interview. Those personally examined underwent a silent count of the radial pulse while the patient simultaneously counted silently the $P$ pulsations. The counts were always within $5 \%$ of each other. Thyroid function tests, hematocrits, auscultation, and otoscopy were normal in all. Except for VC, imaging did not reveal abnormalities to account for $\mathrm{P}$. Six had angiography. Three had normal lumbar punctures. Audiometry was performed in all but was not available for 5 of the remote subjects.

\section{RESULTS}

Audiometry: Pure tone thresholds were normal in 16 subjects and symmetric in 22 subjects. 15 subjects had asymmetric thresholds; for 13 of them their $\mathrm{P}$ was lateralized to the side with the poorer thresholds.

Categorization of cases: The 58 cases were categorized according to whether or not the $P$ is $(A)$ constant, $(B)$ intermittent only, and $(C)$ intermittent together with $\mathrm{nP}$. The general categories can be further divided into more specific subcategories depending upon their response to ST, thereby yielding a total of 8 categories as shown in Table 1 and Figure 1.

A. Constant Pulsatile Tinnitus (Pc): Thirty-nine (67\%) of the cases had constant pulsatile tinnitus that was usually described as high-pitched.

A1. Constant P - suppressed by ST (PCS): $90 \%$ with constant $P$ met the original SSPT criteria: total transient $P$ suppression with ST. 26 of 35 PcS subjects (74\%) could suppress all tinnitus; in nine (26\%) only the pulsatile quality of the tinnitus was suppressed, leaving behind residual high pitched $\mathrm{nP}$. PcS cases were about equally

Table 1: Categorization of cases of pulsatile tinnitus without an identifiable acoustic source (P).

\begin{tabular}{|c|c|c|c|c|}
\hline & Categories of $\mathbf{P}$ & Symbol & \# of cases & $\%$ \\
\hline A1 & Constant P - suppressed by ST & PcS & 35 & 60 \\
\hline A2 & Constant P - NOT suppressed by ST & $\mathrm{PcO}$ & 4 & 7 \\
\hline B1 & Intermittent P - suppressed by ST & PiS & 4 & 7 \\
\hline B2 & Intermittent P - P elicited by ST & $\mathrm{Pi}+$ & 1 & 2 \\
\hline $\mathrm{C} 1$ & Intermittent $\mathrm{P}$ together with $\mathrm{nP}$ - P suppressed by $\mathrm{ST}$ & $\mathrm{nP} / \mathrm{PiS}$ & 4 & 7 \\
\hline $\mathrm{C} 2$ & Intermittent P together with nP - P elicited by ST & $\mathrm{nP} / \mathrm{Pi}+$ & 5 & 9 \\
\hline C3 & Intermittent $\mathrm{P}$ together with $\mathrm{nP}$ - P NOT elicited by ST & $\mathrm{nP} / \mathrm{Pi} \pm$ & 4 & 7 \\
\hline $\mathrm{C} 4$ & Intermittent $\mathrm{P}$ together with $\mathrm{nP}-\mathrm{ST}$ (i) elicited $\mathrm{P}$ and (ii) did NOT suppress $\mathrm{P}$ * & $\mathrm{nP} / \mathrm{Pi}+, 0$ & 1 & 2 \\
\hline
\end{tabular}

*This case of $\mathrm{nP} / \mathrm{Pi}$ was tested twice. At the first encounter when $\mathrm{P}$ was not present ST elicited $\mathrm{P}$. At the second encounter $\mathrm{P}$ was present but ST did not suppress $P$ 


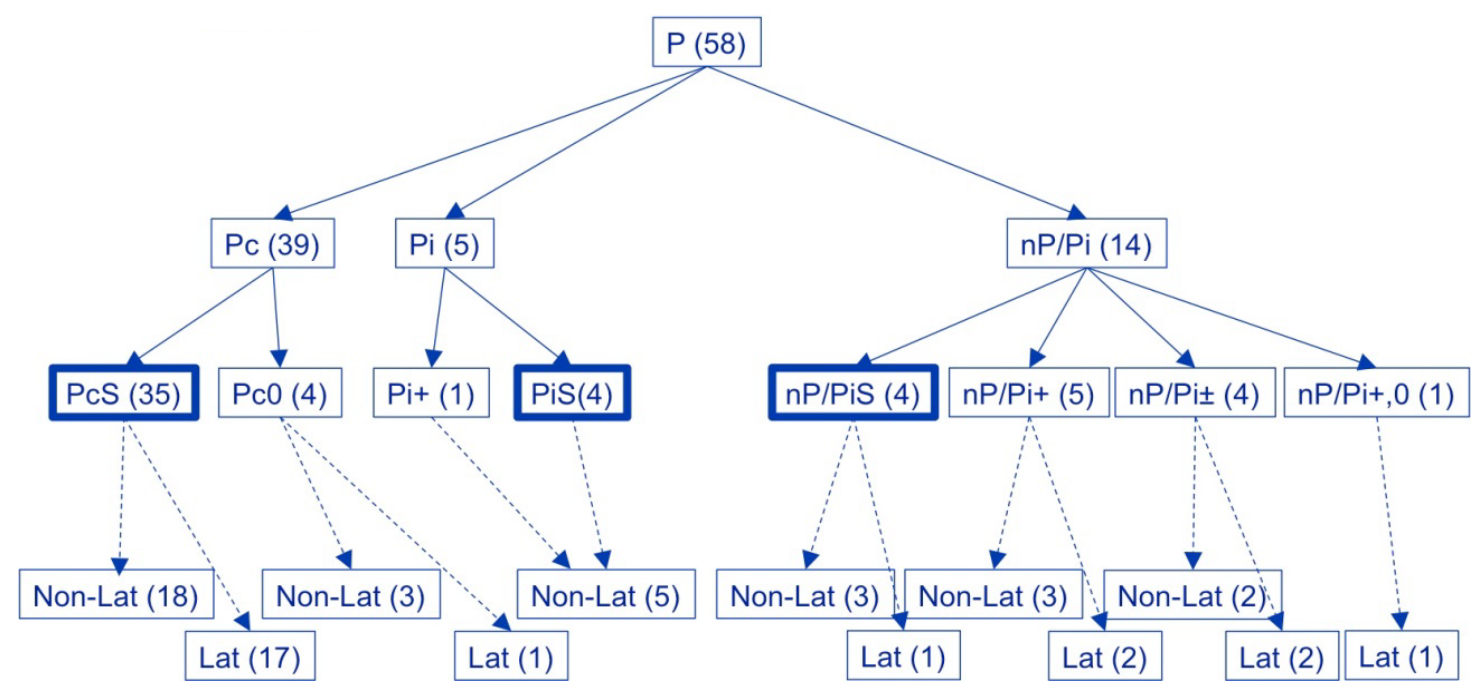

Figure 1: Subtypes of subjective pulsatile tinnitus $(P)$. The number of cases of each subtype is shown within the parentheses next to its symbol. The 4th and 5th rows show the number of cases within each subtype whose $\mathrm{P}$ is non-lateralized (Non-Lat) or lateralized (Lat). $\mathrm{Pc}=$ constant $\mathrm{P} ; \mathrm{PcS}=\mathrm{Pc}$ whose pulsations could be suppressed. $\mathrm{Pc0}=\mathrm{Pc}$ whose pulsations could not be suppressed; $\mathrm{Pi}$ $=$ intermittent $\mathrm{P} ; \mathrm{PiS}=\mathrm{Pi}$ whose pulsations could be suppressed; $\mathrm{Pi}+=\mathrm{Pi}$ whose $\mathrm{P}$ was not present at time of testing but $\mathrm{P}$ was elicited by $\mathrm{ST} ; \mathrm{nP} / \mathrm{Pi}=$ both non-pulsatile tinnitus and $\mathrm{Pi} ; \mathrm{nP} / \mathrm{PiS}=\mathrm{nP} / \mathrm{Pi}$ whose pulsations could be suppressed; $\mathrm{nP} / \mathrm{Pi}+=\mathrm{nP} / \mathrm{Pi}$ whose $\mathrm{P}$ was not present at time of testing but $\mathrm{P}$ was elicited by $\mathrm{ST} ; \mathrm{nP} / \mathrm{Pi} \pm=n P / \mathrm{Pi}$ whose $\mathrm{P}$ was not present at time of testing and was not elicited by $\mathrm{ST} ; \mathrm{nP} / \mathrm{Pi}+, 0=$ the unique case whose $\mathrm{P}$ was elicited at her first visit and at her next visit $\mathrm{P}$ was present but could not be suppressed

split between those with lateralized $\mathrm{P}(17)$ and those with non-lateralized $\mathrm{P}(18)$ as shown in Figure 1.

A2. Constant $\mathbf{P}$ not suppressed by ST (Pc0): While similar in all other respects to the PcS group, the four Pc0 patients could not totally suppress their pulsations with ST but all could modulate their Pc0 with ST. Three of the four had non-lateralized Pc0. The one subject with left Pc0 elicited right Pc0 with ST. A second Pc0 subject reported a new $\mathrm{nP}$ and non-lateralized tinnitus with ST. Two described their Pc0 as high-pitched, another medium-pitched and the fourth subject was unable to estimate the dominant pitch. Overall with ST the Pc0 loudness modulated only louder in two and both quieter and louder in two.

B. Intermittent Pulsatile Tinnitus (Pi) Only: Five subjects had $\mathrm{Pi}$ only. All were non-lateralized; their $\mathrm{P}$ was usually heard daily.

B1. Intermittent P - suppressed by ST (PiS): All four, who were hearing their $\mathrm{P}$ when examined, could abolish it with ST.

B2. Intermittent $\mathbf{P}$ - $\mathbf{P}$ elicited by $\mathbf{S T}(\mathbf{P i}+)$ : The one subject, who was not having her intermittent $P$ at the time of testing, elicited her P with ST.

Recumbent Pulsatile Tinnitus: Four of the five with intermittent $P$ only reported a close relationship between their intermittent $P$ and recumbency, as did another two who also heard $\mathrm{nP}$ in addition to their intermittent $\mathrm{P}$ (Table 2). Five of the six were women with non-lateralized $P$. The sixth was a man $(M / 81)$ with lateralized intermittent $P$. For five their intermittent $P$ occurred almost exclusively with prolonged recumbency and disappeared upon arising from bed. $F / 40$ at times did not hear her $P$ when recumbent, but always upon awakening, even if not heard overnight. Only F/70 was examined when recumbent and having $P$.

Case 1(F/70): Recumbent PiS. She reported having 7 months of non-lateralized intermittent $P$ ("motor") that occurred daily and almost exclusively after recumbent in bed for several minutes. Audiogram, MRA and MRV were normal. At her initial visit her $P$ was not present despite being supine for about five minutes. No bruits were detected. ST elicited no tinnitus. At her next visit after lying awake and supine for 30 minutes, her $\mathrm{P}$ began localized to her occiput. Her silent 30-second count of her pulsations and the examiner's simultaneous count of her radial pulse were identical. No bruits were detected. Right, left, or bilateral jugular compression did not alter her $P$. Active turning of her head to the left increased the loudness of her $P$. Her $P$ was unchanged by active or passive neck forward flexion, but her $\mathrm{P}$ was abolished by neck forward flexion against resistance. When contacted five years later her "recumbent" PiS rarely occurred.

C. Intermittent $\mathbf{P}$ together with $\mathrm{nP}(\mathrm{nP} / \mathrm{Pi})$ : Fourteen (24\%) had $\mathrm{nP}$ in addition to intermittent $\mathrm{P}$. This group is diverse. In all the $\mathrm{nP}$ was more frequent than the $\mathrm{P}$. The $\mathrm{nP}$ was constant in nearly all. The major exception was $\mathrm{M} / 81$ (Table 2). The localization of the $\mathrm{nP}$ and intermittent $P$ was the same for all subjects, except one. This subject had had 9 months of non-lateralized $\mathrm{nP}$ before she began treating with a chiropractor. During the 3 weeks of these treatments, she developed left intermittent $P$; she then stopped all treatments and within another 3 weeks her intermittent $\mathrm{P}$ stopped. 
Table 2: Six cases with $\mathrm{P}$ closely related to recumbency.

$\begin{array}{cccc}\text { Sex/Age } & \text { Lat? } & \text { Relationship to recumbency } & \mathrm{P} \text { Subtype } \\ \mathrm{F} / 62 & \text { No } & \text { Primarily in bed. Stops immediately when sits up } & \mathrm{Pi}+ \\ \mathrm{F} / 70 & \text { No } & \text { Hear in bed almost exclusively } & \mathrm{PiS} \\ \mathrm{F} / 47 & \text { No } & \mathrm{P} \text { always in bed before arising; and again late in day } & \mathrm{PiS} \\ \mathrm{F} / 40 & \text { No } & \mathrm{P} \text { always upon awakening. Gradually quiets during day } & \mathrm{PiS} \\ \mathrm{F} / 48 & \text { No } & \text { Always } \mathrm{P} \text { in bed but not upon awakening } & \mathrm{nP} / \mathrm{Pi}+ \\ \mathrm{M} / 81 & \text { Yes } & \mathrm{P} \text { only after recumbent 5-10 minutes } & \mathrm{nP} / \mathrm{Pi} \pm\end{array}$

Lat? = whether or not $\mathrm{P}$ was lateralized.

Eight described their $\mathrm{nP}$ as non-lateralized and six lateralized. For five of this group, ST suppressed their nP (2 lateralized, 3 non-lateralized).

C1. Intermittent $P$ together with $\mathrm{nP}$ - $\mathrm{P}$ suppressed by ST (nP/PiS): Like the Pi subjects, the four nP/Pi subjects, who were tested with ST just once and had $P$ at the time of testing, could abolish it (nP/PiS).

C2. Intermittent $P$ together with $\mathrm{nP}$ - $\mathrm{P}$ elicited by ST $(\mathbf{n P} / \mathbf{P i}+)$ : Five subjects with $\mathrm{nP} / \mathrm{Pi}$ were tested with ST just once and had no $\mathrm{P}$ immediately prior to ST, but ST elicited their $P$.

C3. Intermittent $\mathbf{P}$ together with $\mathrm{nP}$ - $\mathrm{P}$ NOT elicited by $\mathbf{S T}(\mathrm{nP} / \mathbf{P i} \pm)$ : Another four subjects with $\mathrm{nP} / \mathrm{Pi}$, who underwent ST just once and had no $P$ immediately prior to ST, did not elicit their $\mathrm{P}$ with ST.

C4. Intermittent $\mathbf{P}$ together with $\mathrm{nP}$ - tested twice with ST: This subject was unique. At her initial visit her $P$ was not present but was elicited by ST. At her second visit $\mathrm{P}$ was present but could not be suppressed (hence $\mathrm{nP}$ / $\mathrm{Pi}+, 0)$.

\section{DISCUSSION}

Onceitwas recognized thatactivation of the somatosensory system of the head and neck can have a major influence upon auditory perception and in particular tinnitus ${ }^{8,9}$, routine ST in the evaluation of tinnitus patients has been advocated to identify tinnitus subgroups amenable to specific treatments ${ }^{10}$. Hence finding that ST could suppress $P$ was not entirely serendipitous. Our original paper reported upon thirteen consecutive $P$ patients seen over $2-1 / 2$ years; this subgroup was referred to as SSPT. Their common features were constant $P$ whose pulsations could be transiently suppressed by ST. To maintain a consistent terminology encompassing other $P$ variants, $\mathrm{PcS}$ as used herein is synonymous with the original SSPT subjects. In the original report the 13 PcS were about equally split between lateralized and non-lateralized, just as in the $35 \mathrm{PcS}$ cases in this report. The previous report also found that ST abolished all tinnitus in $70 \%$ and only the pulsatile quality in $30 \%$. The comparable figures for the present study are very similar, $74 \%$ and $26 \%$. Also in our previous study one case of $\mathrm{nP} / \mathrm{PiS}$ was identified?.

While in general $80 \%$ of people with tinnitus can alter its perception with ST, only $14 \%$ can abolish it ${ }^{8}$. This contrasts to $67 \%$ with $\mathrm{Pc}$ who can abolish $\mathrm{P}$ in the present study and $74 \%$ previously. Because of this highly significant difference it was argued previously for a somatosensoryrelated mechanism causing PcS. Two were proposed. Both involved a modification of the original somatic tinnitus theory that hypothesized that tinnitus results from head and neck muscle afferents modifying the activity of the ipsilateral dorsal cochlear nucleus (DCN) via the trigeminal-cervical-complex ${ }^{11}$. One suggested that the neural activity of head and neck somatosensory afferents to the auditory CNS was cardiac synchronous and thereby caused $\mathrm{P}$. The second proposed that head and neck somatosensory afferents via the above CNS circuitry cause a disruption of one of the functions of the DCN. That DCN function is the suppression of self-generated sounds including heart beats ${ }^{12}$.

Non-lateralized P: After considering several possibilities to account for non-lateralized $P$, it was previously concluded that the only viable hypotheses were cardiac synchronous modulation of the auditory CNS either (a) in one locus rostral to the trapezoid body or (b) in two loci caudal to the trapezoid body such as interactions between neural structures from both sides of the brainstem, e.g. through the reciprocal connections between the right and left cochlear nuclei ${ }^{7,13}$.

Additional support for the cochlear nuclei involvement in $P$ comes from one of the present cases, a subject with non-lateralized PcS, which was described as louder on the right. At angiography amobarbital was injected into the "distal right anterior inferior cerebellar artery beyond the anterior and lateral brainstem" branches. His right $P$ was abolished for five minutes but his left remained unchanged. Presumably this effect of amobarbital was from suppressing neural activity arising from his right cochlear nucleus ${ }^{14}$.

Four other subjects had negative cerebral angiography. Two of these underwent temporary balloon occlusion of multiple arteries and veins with no alteration of their $P$. No others received amobarbital.

\section{New Categories of $\mathbf{P}$ not previously reported}

Our observations of $P$ subsequent to our first report now include six new categories not previously described, A2, $\mathrm{B} 1, \mathrm{~B} 2, \mathrm{C} 2, \mathrm{C} 3$, and $\mathrm{C} 4$. Our original report described the major category PcS and one other case of $\mathrm{nP} / \mathrm{PiS}$. The new categories account for 24 of the 58 (41\%) subjects with $\mathrm{P}$ encountered since our first report.

Of the five subjects who could not suppress their $P$ (the four $\mathrm{PcO}$ subjects and the one $\mathrm{nP} / \mathrm{Pi}+, 0$ subject), two 
elicited $\mathrm{P}$ with ST, which suggests a close relationship between their $\mathrm{P}$ and the somatosensory system. In the one case with $\mathrm{Pc0}$, her $\mathrm{Pc}$ was lateralized to the left, and her elicited $P$ was on the right, contralateral to her ongoing Pc. The other was a case with $\mathrm{nP} / \mathrm{Pi}$ who was tested with ST twice, once with and once without her $\mathrm{Pi}$ (C4 of Table 1).

Similarly the other six intermittent $\mathrm{P}$ cases (one $\mathrm{Pi}+$ and five $\mathrm{nP} / \mathrm{Pi}+$ ), whose $\mathrm{P}$ was not present at the time of testing but was elicited by ST, also suggests a close relationship between their $\mathrm{P}$ and the somatosensory system. What is likely accounting for the $\mathrm{Pi}+$ and $\mathrm{nP} / \mathrm{Pi}+$, cases is as follows. In general with the maneuvers of $\mathrm{ST}$, tinnitus can be modulated louder or softer. In these $\mathrm{Pi}+$ subjects their $\mathrm{Pi}$ is not present at the time of testing. For this reason only the maneuvers that cause louder $\mathrm{P}$ will be detected thereby being classified as $\mathrm{Pi}+$. Maneuvers that cause suppression of $P$ will not be detected, since there is no ongoing $P$ to suppress ${ }^{15}$.

Case in point is the patient described in our previous report. From her exam she was a $\mathrm{nP} / \mathrm{Pi}+$ since she had no ongoing $\mathrm{P}$ at time of testing but did elicit her $\mathrm{P}$ with ST. However she related that, when present, she could suppress her left $P$ with right jaw deviation; hence in our current classification system she went from a $\mathrm{nP} / \mathrm{Pi}+$ to $\mathrm{nP} / \mathrm{PiS}^{7}$.

In addition in one of our recent $\mathrm{nP} / \mathrm{Pi}$ cases, at the start of ST she had $n P$ but no P. About half way through ST, P was elicited; with continued ST she could abolish both her elicited $\mathrm{P}$ as well as her $\mathrm{nP}$. She was ultimately classified as $\mathrm{nP} / \mathrm{PiS}$.

It is unknown whether the $\mathrm{P}$ of the other cases of $\mathrm{Pi}+$ and $\mathrm{nP} / \mathrm{Pi}+$ would fall into the $\mathrm{PiS}, \mathrm{nP} / \mathrm{PiS}$ or $\mathrm{nP} / \mathrm{Pi}+, 0$ categories, if their $\mathrm{P}$ had been present when they underwent ST.

Regarding the four $\mathrm{nP} / \mathrm{Pi} \pm$ cases, none were retested while their $\mathrm{P}$ was present. As described above, one of the two with lateralized $\mathrm{Pi}$ had her $\mathrm{P}$ associated with cervical chiropractic manipulation. This association strongly suggests a somatic basis for her $\mathrm{Pi}$. The recumbent $\mathrm{P}$ case presented above (case 1) is also supportive of a somatic basis for the $\mathrm{nP} / \mathrm{Pi} \pm$ cases, since at her first visit ST did not elicit $P$ so she would have been classified as $\mathrm{Pi} \pm$. However at her repeat visit her $\mathrm{P}$ was present and abolished by ST. Her second ST reclassified her from $\mathrm{Pi} \pm$ to PiS.

RECUMBENT P: The association between recumbency and $P$ (Table 2 and case 1) again is supportive of the somatosensory factor and $P$. As observed for case 1 , her $P$ was not related to sleep but rather to recumbency, since her $P$ appeared after 30 minutes and yet she was awake. With recumbency a major change occurs in the state of the cervical muscles, tendons and joints. When upright these cervical structures are supporting the 10-pound head but, when recumbent, these same structures are in a more relaxed state. In these six cases it is likely that the change in the state of the cervical muscles, tendons and joints is responsible for the change in $P$.

IS VC ACCOUNTING FOR P?: While VC cannot account for non-lateralized $P$, as the only well-established cause for lateralized P, VC may be accounting for some cases of lateralized $P$. Because surgically proven VC cases of $P$ have not been tested with ST, there may be an overlap between lateralized P and VC. One of our 24 lateralized cases documents that this can be the case.

Case 2: A 60-year-old woman reported at her first visit that her right ear tinnitus began 3 months earlier as a few occurrences of a "foghorn" lasting a few seconds; a week later it became constant and cardiac synchronous. When her tinnitus was very quiet, she heard only pulsatile "clicking," when loud only pulsatile "whooshing." No bruits were detected in the cervical or periauricular regions. Jugular compression did not alter her tinnitus. With ST, all tinnitus was abolished by resisted neck forward flexion. Her audiogram showed normal pure tone thresholds at 3 $\mathrm{kHz}$ and below. Above $3 \mathrm{kHz}$ was a sloping loss reaching $40 \mathrm{~dB}$ at $8 \mathrm{kHz}$ for both ears. Thresholds were identical at the two ears except for $15 \mathrm{~dB}$ poorer for the right ear at $1 \mathrm{kHz}$ and $3 \mathrm{kHz}$. Her CISS MRI scan detected VC

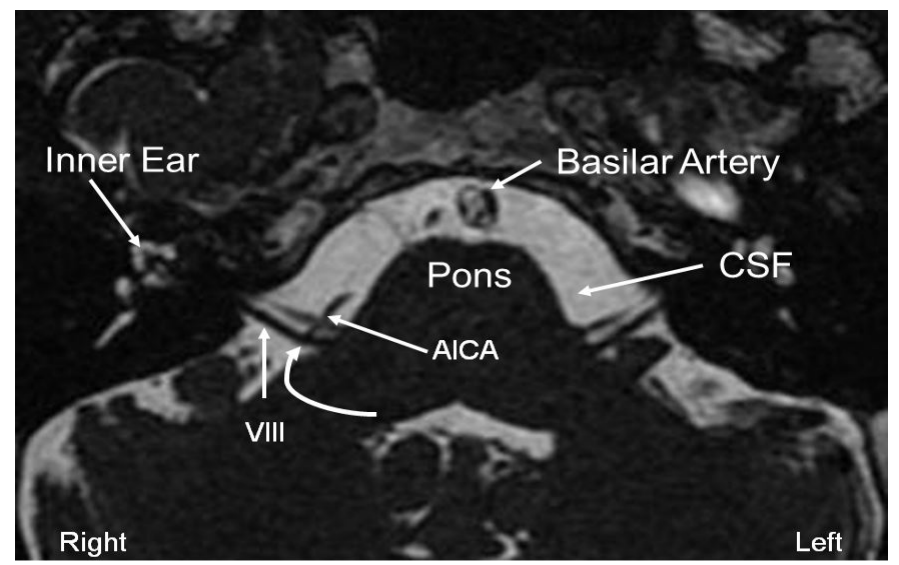

Figure 2: MRI scan showing vascular compression (VC) of right VIIlth nerve of Case 2 by the right anterior inferior cerebellar artery (AICA). The point of contact between AICA and right VIIIth nerve (curved arrow) results in deviation of the VIIlth nerve from the normal straight path, as can be seen for the contralateral VIIIth nerve. CSF = cerebrospinal fluid. From Levine RA, Oron Y. Tinnitus ${ }^{15}$. 
of the right VIIlth nerve in its cisternal segment. (Figure 2). A trial of carbamazepine was aborted after 3 days due to a rash. Within less than a year from its onset her clicking had stopped. Presently (nine years from onset) whooshing PcS persists.

The presence of VC on MRI alone does not implicate VC as the etiology of her $\mathrm{P}$, since many patients have this finding with no symptoms. However, when the MRI finding is taken together with clicking which has been shown to be pathognomonic for $\mathrm{VC}$, then $\mathrm{VC}$ as the etiology of her lateralized PcS becomes unquestionable ${ }^{16-18}$. In addition her $\mathrm{P}$ is low pitched as has been reported for cases of surgically verified $\mathrm{VC}^{3}$. Another one of our cases of lateralized PcS was felt by the radiologist to have a vascular loop within his left internal auditory canal "where there may be neurovascular contact." The patient described his $\mathrm{P}$ as constant, left and high-pitched; it was abolished by right jaw deviation against resistance. A third subject $(\mathrm{M} / 81$ of Table 2$)$ with lateralized $\mathrm{nP} / \mathrm{Pi}$ heard his left low-pitched $P$ only when recumbent; he was never tested while having his P. His MRI scan was highly suspicious for vascular compression at his left auditory nerve root entry zone.

In all three of these cases the P was "fully lateralized". It was perceived in the ear itself, not in the vicinity of the ear or the side of the head and they reported no tinnitus in the contralateral ear. From case 2 with definite VC, when taken together with the two other cases with imaging suggestive of VC, it suggests that $P$ from VC (a) is "fully lateralized" (perceived in the ear itself) and (b) can be abolished by ST. It is an identifiable cause of lateralized PcS and possibly of lateralized intermittent $P$ with or without $\mathrm{nP}$.

The fact that $P$ from VC can be suppressed by ST raises questions about the mechanism of suppression. Since (a) the tinnitus of VC presumably arises from the auditory nerve itself and (b) cervical and head somatosensory afferents do not project directly or indirectly to the nerve but to the DCN, these two facts suggest that the suppression of $P$ from $V C$ is occurring at the $D C N$.

Response to Treatment: In four of our cases there has been a major response to treatment. While anecdotal they have implications for possible mechanisms underlying $P$ that is either not related to VC or combines with VC to cause $P$.

\section{Dry needling of cervical trigger points}

Case 3: About three times a year over four years, a 70-year-old otolaryngologist would develop severe rightsided neck pain and right-sided $P$. Typically after 3 sessions of dry needling of his right sternocleidomastoid and adjacent muscles, the $\mathrm{P}$ and pain resolved.

Case 4: A 67-year-old woman developed non-lateralized $\mathrm{nP}$ together with constant $\mathrm{P}$ that was usually but not always left sided. Her exam revealed multiple left posterolateral cervical trigger points. Seven months after the onset of her $P$, she received four sessions of dry needling of her cervical trigger points within a span of six weeks, after which her $\mathrm{P}$ resolved but not her $\mathrm{nP}$.

Botulinum toxin injections of cervical trigger points: A colleague has reported two patients whose $P$ resolved with botulinum toxin injections of splenius capitis trigger points located at the craniocervical junction. ${ }^{19}$

Auricular electrical stimulation device (AESD: 3 days of continuous 1 per sec, 1 msec pulses) ${ }^{20}$

Case 5: A 58-year-old woman had had right-sided PcS for one year; with ST the pulsatile quality of her P could be transiently totally suppressed. Following 7 weekly applications of AESD, her PcS continued to attenuate and by four months she had no tinnitus of any kind.

Case 6: After 10 weekly AESD applications for nonlateralized PcS that had been present for more than a year, this 59-year-old man's $P$ stopped and was replaced by intermittent $\mathrm{nP}$ that was not heard about a third of the time and was quieter than his PcS had been. The benefit presently has persisted more than four years.

The vagus, trigeminal and upper cervical spinal nerves provide the sensory innervation of the auricle ${ }^{21}$. All three nerves send projections to a common region of the lower medulla, known as the medullary somatosensory nucleus, a subunit of the trigeminal-cervical-complex, from which fibers project to the ipsilateral DCN. In this way these AESD results, as well as the dry needling results, can be understood as impacting upon the auditory pathway via the CNS somatosensory-auditory interactions.

\section{CONCLUSION}

This sequel to our earlier report of the SSPT syndrome has expanded upon the initial observations. While the dominant manifestation of $P$ is as before $\mathrm{PcS}$, other types occur. (1) P can be intermittent with or without $\mathrm{nP}$. (2) In about $10 \%$ of cases pulsations are not ST-suppressible. Whether or not the pulsations can be suppressed by ST, non-lateralized $P$ continues to be best explained as before from either (A) failure of the somatosensory-auditory CNS interactions to suppress cardiac somatosounds, (B) cardiac synchronous somatosensory activation of the central auditory pathway or $(C)$ some combination of $A$ and $\mathrm{B}$.

Our observation that lateralized PcS can occur in one definite case of $P$ with $V C$ (and possibly more) raises the possibility that not all lateralized $P$, even if the pulsations can be somatically suppressed, is solely somatosensory in origin but to some degree may be related to ipsilateral VC. Our observations suggest that, if the $P$ is perceived as "fully lateralized," i.e. coming from the ear itself, then a $\mathrm{VC}$ contribution to the $\mathrm{P}$ is more likely. If $\mathrm{VC}$ is contributing to $P$, vascular decompression appears to have a high likelihood of abolishing the $P$.

Resolution of $\mathrm{P}$ by AESD or by dry needling and botulinum toxin injections of cervical musculature substantiates that 
the pulsatile tinnitus is not a somatosound. This report provides further support to the concept that $P$ is intimately related to the cervical somatosensory system, based upon (A) the eliciting of $P$ by ST in cases without $P$ at the time of $S T,(B)$ the response of $P$ to both dry needling and botulinum toxin injections and $(\mathrm{C})$ the association of $\mathrm{Pi}$ with recumbency.

\section{CONFLICT OF INTEREST}

The authors declare no potential conflict of interest

\section{REFERENCES}

1. Song JJ, An GS, Choi I, Ridder DD, Kim Sy, Choi HS, et al. Objectification and differential diagnosis of vascular pulsatile tinnitus by transcanal sound recording and spectrotemporal analysis: A preliminary study. Otol Neurotol. 2016;37:61320.

2. Ohashi N, Yasumura S, Nakagawa H, Mizukoshi K, Kuze S. Vascular cross-compression of the VIlth and VIIIth cranial nerves. J Laryngol Otol. 1992;22:436-39.

3. Ryu H, Yamamoto S, Sugiyama K, Uemura K, Nozue M. Neurovascular decompression of the eighth cranial nerve in patients with hemifacial spasm and incidental tinnitus: An alternative way to study tinnitus. J Neurosurg. 1998;88:23236.

4. Vasama JPI, Moller MB, Moller AR. Microvascular decompression of the cochlear nerve in patients with severe tinnitus. Preoperative findings and operative outcome in 22 patients. Neurol Res. 1998;20:242-48.

5. Mathiesen T, Brantberg K. Microvascular decompression for typewriter tinnitus-case report. Acta Neurochir (Wien). 2015;157:333-6.

6. Levine RA. Typewriter tinnitus: A carbamazepine-responsive syndrome related to auditory nerve vascular compression. ORL. 2006;68:43-7.

7. Levine RA, Nam E-C, Melcher J. Somatosensory Pulsatile Tinnitus Syndrome: Somatic Testing Identifies a Pulsatile Tinnitus Subtype That Implicates the Somatosensory System. Trends Amplif. 2008;12:242-53.

8. Levine RA, Abel M, Cheng H. CNS somatosensory-auditory interactions elicit or modulate tinnitus. Exp Brain Res. 2003;153:643-48.
9. Lewald J, Karnath HO, Ehrenstein WH. Neck-proprioceptive influence on auditory lateralization. Exp Brain Res. 1999;125:389-96.

10. Levine RA, Nam EC, Oron Y, Melcher JR. Evidence for a Tinnitus Subgroup Responsive to Somatosensory Based Treatment Modalities.brain research. 2007:195-207.

11. Levine RA. Somatic (craniocervical) tinnitus and the dorsal cochlear nucleus hypothesis. Am J Otolaryngol - Head Neck Med Surg. 1999;20:351-62.

12. Haenggeli CA, Pongstaporn T, Doucet JR, Ryugo DK. Projections from the spinal trigeminal nucleus to the cochlear nucleus in the rat. J Comp Neurol. 2005;484:191-205.

13. Cant NB, Gaston KC. Pathways connecting the right and left cochlear nuclei. J Comp Neurol. 1982;212:313-326.

14. De Ridder D, Fransen H, Francois O, Sunaert S, Kovacs S, Van De Heyning P. Amygdalohippocampal involvement in tinnitus and auditory memory. In: Acta Otolaryngol Suppl. 2006;123:50-53.

15. Levine RA, Oron Y. Tinnitus. In: Handbook of Clinical Neurology. 2015;409-31.

16. Brantberg K. Paroxysmal staccato tinnitus: A carbamazepine responsive hyperactivity dysfunction symptom of the eighth cranial nerve. J Neurol Neurosurg Psychiatry. 2010;81:45155.

17. Sunwoo W, Jeon YJ, Bae YJ, Jang JH, Koo JW, Song JJ. Typewriter tinnitus revisited: The typical symptoms and the initial response to carbamazepine are the most reliable diagnostic clues. Sci Rep. 2017;7:106-15.

18. Bae YJ, Jeon YJ, Choi BS, Koo JW, Song JJ. The role of MRI in diagnosing neurovascular compression of the cochlear nerve resulting in typewriter tinnitus. Am $\mathrm{J}$ Neuroradiol. 2017;38:1212-17.

19. Ranoux D. Onabotulinumtoxin A. Injections of Splenius Capitis in Chronic Migraine Abolishes Pulsatile Tinnitus. 2020.

20. Cardarelli F, Melcher J, Szeles J, Levine RA. Continuous auricular electrical stimulation quiets the tinnitus of the somatosensory pulsatile tinnitus syndrome. In: Frontiers in Tinnitus Research: Fourth International TRI Tinnitus Conference, Dallas, Texas. June. 2010.

21. Peuker ET, Filler TJ. The nerve supply of the human auricle. Clin Anat. 2002;15:35-7. 